\title{
Magnetohydrodynamic waves in a non-uniform current-carrying plasma column
}

\author{
By J. VACLAVIK AND E. WEISE \\ Department of Physics, University of Fribourg, Switzerland
}

(Received 11 February 1974)

An effect of an axial current on the propagation of low-frequency axisymmetric magnetohydrodynamic waves in a radially non-uniform plasma column was investigated theoretically and experimentally. It was found that the axial current and the density gradient cause a coupling between the torsional and compressional waves.

\section{Introduction}

The propagation of low-frequency magnetohydrodynamic waves in a nonuniform plasma column has recently been the subject of numerous investigations, both theoretical and experimental. The first theoretical investigation was carried out by Pneuman (1965). Since then, there have been many others (McPherson \& Pridmore-Brown 1966; Cross \& Lehane 1968; Davies 1969; Morrow \& Brennan 1971).

Under the usual laboratory conditions, where the gas pressure is negligible compared with the magnetic pressure, there are just two waves that can propagate at frequencies below the ion cyclotron frequency; these are usually called the torsional (or Alfvén) wave and the compressional (or fast magneto-acoustic) wave. Experimental observations of the axisymmetric torsional and compressional waves, propagating in a radially non-uniform plasma, were reported by Cross \& Lehane (1968), Morrow \& Brennan (1971) and Müller, Räuchle \& Schüller (1973). The non-axisymmetric waves were investigated by McPherson \& Pridmore-Brown (1966), who found that the density gradient introduces a coupling between the torsional and compressional waves. As a result, an axial component of the magnetic field perturbation, which is normally associated only with the compressional wave, is now carried by the torsional wave as well, and was in fact detected at frequencies well below the lowest waveguide cut-off of the compressional wave.

In this paper, we investigate an effect of an axial current on the propagation of low-frequency axisymmetric waves in a radially non-uniform plasma column. The plasma is immersed in a uniform axial magnetic field, and the region outside the plasma is assumed to be a vacuum. It turns out that, even in the axisymmetric case, the axial current and the density gradient cause a coupling between the torsional and compressional waves. This coupling is similar to that of the non-axisymmetric waves propagating in a non-uniform plasma with no current (McPherson \& Pridmore-Brown 1966). 
In $\S 2$ a simple theory of the phenomenon to be investigated is developed, assuming that the plasma is fully ionized and ideally conducting. A description of the experimental apparatus and a presentation of the experimental results are given in $\$ 3$. Measurements of signal amplitudes and wave speeds are there discussed in relation to the theory.

\section{Theory}

Let $\rho_{0}, p_{0}, \mathbf{B}_{0}$ represent the equilibrium values of the mass density, pressure and magnetic field. The linearized equations of ideal single-fluid magnetohydrodynamics can then be written in the form (Kadomtsev 1966)

$$
\begin{gathered}
\rho_{0} \frac{\partial \mathbf{v}}{\partial t}+\nabla p=\frac{1}{4 \pi}\left[\operatorname{curl} \mathbf{B}_{0}, \mathbf{B}\right]+\frac{1}{4 \pi}\left[\operatorname{curl} \mathbf{B}, \mathbf{B}_{0}\right] \\
\frac{\partial p}{\partial t}+(\mathbf{v} . \nabla) p_{0}+\gamma p_{0} \operatorname{div} \mathbf{v}=0 \\
\frac{\partial \mathbf{B}}{\partial t}=\operatorname{curl}\left[\mathbf{v}, \mathbf{B}_{0}\right]
\end{gathered}
$$

where $\mathrm{v}$ is the plasma velocity and $\gamma$ the adiabaticity index.

In what follows, we adopt a cylindrical co-ordinate system $r, \phi, z$, and assume that $\partial \partial \phi \equiv 0$ throughout. For the equilibrium quantities of the plasma, we can then use the simplified model of pinched plasma column. Thus,

$$
\left.\begin{array}{rl}
\mathbf{B}_{0} & =\left(0, B_{0 \phi}(r), B_{0 z}\right), \\
\frac{d p_{0}}{d r} & =-\frac{B_{0 \phi}}{4 \pi} \frac{1}{r} \frac{d}{d r}\left(r B_{0 \phi}\right) \quad(r \leqslant R), \\
p_{0} & =0 \quad(r \geqslant R),
\end{array}\right\}
$$

where $R$ is the radius of the column.

For the first-order quantitaties $\mathbf{v}, p, \mathbf{B}$, we next introduce the ansatz

$$
\exp \{i(k z-\omega t)\}
$$

in (1)-(3). Since the quantities of interest in the measurements are the magnetic field components $B_{r}, B_{\phi}, B_{z}$ we eliminate the other variables in favour of them. On making use of (4), (1)-(3) can then be reduced to one equation involving the quantity $B_{r}$. Moreover, if we assume, in agreement with the experimental data, that

we obtain the wave equation

$$
4 \pi \gamma p_{0} / B_{0}^{2} \ll 1
$$

$$
\frac{d}{d r}\left(\frac{N}{D} r \frac{d B_{r}}{d r}\right)+\left\{\frac{k^{2}}{r} \frac{d}{d r}\left(r \frac{C_{\phi}^{2}}{C_{z}^{2} D}\right)+\frac{1}{r}\left[\left(\frac{C_{\phi}}{C_{z}}\right)^{2}-1\right]-r N\right\} B_{r}=0
$$

where

$$
\begin{gathered}
N=k^{2}-\left(\frac{\omega}{C_{z}}\right)^{2}, \quad D=k^{2}-\left(\frac{\omega}{C_{A}}\right)^{2}, \\
C_{A}^{2}=C_{z}^{2}+C_{\phi}^{2}, \quad C_{z}^{2}=\frac{B_{0 z}^{2}}{4 \pi \rho_{0}}, \quad C_{\phi}^{2}=\frac{B_{0 \phi}^{2}}{4 \pi \rho_{0}} .
\end{gathered}
$$


The quantities $B_{\varepsilon}$ and $B_{\phi}$ are expressed explicitly in terms of $B_{r}$ as

$$
\begin{gathered}
B_{z}=\frac{i}{k} \frac{1}{r} \frac{d}{d r}\left(r B_{r}\right), \\
B_{\phi}=\frac{i}{B_{0 z} D}\left\{\frac{k}{r} \frac{d}{d r}\left(r B_{0 \phi}\right) B_{r}-\frac{1}{k}\left(\frac{\omega}{C_{A}}\right)^{2} \frac{d}{d r}\left(B_{r} B_{0 \phi}\right)\right\} .
\end{gathered}
$$

Finally, (5) must be supplemented by boundary conditions. Consistently with (4), we assume that no current flows at the plasma-vacuum interface. The appropriate boundary conditions are then given by the requirement that $B_{r}$ and $d B_{r} / d r$ are continuous across the plasma-vacuum interface at $r=R$ (Kadomtsev $1966)$ and $B_{r}$ vanishes at infinity.

Now, if the equilibrium current were absent, so that $B_{0 \phi}=0$, we should immediately obtain two uncoupled waves from (5) and (7):

$$
\begin{gathered}
\frac{d}{d r}\left(r \frac{d B_{r}}{d r}\right)-\left(\frac{1}{r}+r N\right) B_{r}=0 \\
N B_{\phi}=0 .
\end{gathered}
$$

These equations describe, respectively, the compressional and torsional waves. The compressional wave will exhibit a cut-off frequency, below which only the torsional wave should be found.

In general, (5) cannot be solved in terms of elementary functions; but, if the functions $B_{0 \phi}(r)$ and $\rho_{0}(r)$ are given, it is easy to get solutions by numerical integration. The $B_{r}, B_{z}$ and $B_{\phi}$ profiles, together with the corresponding eigenvalues of $k$ and the value of the lowest cut-off frequency, were computed for the experimentally determined functions $B_{0 \phi}(r)$ and $\rho_{0}(r)$. The results are presented in $\S 3$. From these computations, we conclude that, even in the case when $B_{0 \phi} \neq 0$, (5) possesses a discrete spectrum of eigenvalues of $k$ at all frequencies above a lowest cut-off frequency. We continue to associate these eigenvalues with the compressional wave.

We now show that, in addition to a discrete spectrum, (5) possesses a continuous spectrum of eigenvalues,

$$
\left(\frac{\omega}{C_{z}}\right)_{\min } \leqslant k \leqslant\left(\frac{\omega}{C_{z}}\right)_{\max },
$$

at all frequencies down to zero. We associate these eigenvalues with the torsional wave. The existence of modes corresponding to these eigenvalues is allowed by the singularity $N\left(r=r^{\prime}\right)=0$ of (5). If we suppose that this is a regular singularity, we find that two independent solutions have series expansions of the form

$$
\left.\begin{array}{l}
B_{r 1}=1+O\left(r-r^{\prime}\right), \\
B_{r 2}=r-r^{\prime}+O\left(\log \left(r-r^{\prime}\right)\right) .
\end{array}\right\}
$$

Thus, we can construct a singular solution which satisfies the boundary conditions by superposing different linear combinations of these independent solutions to 


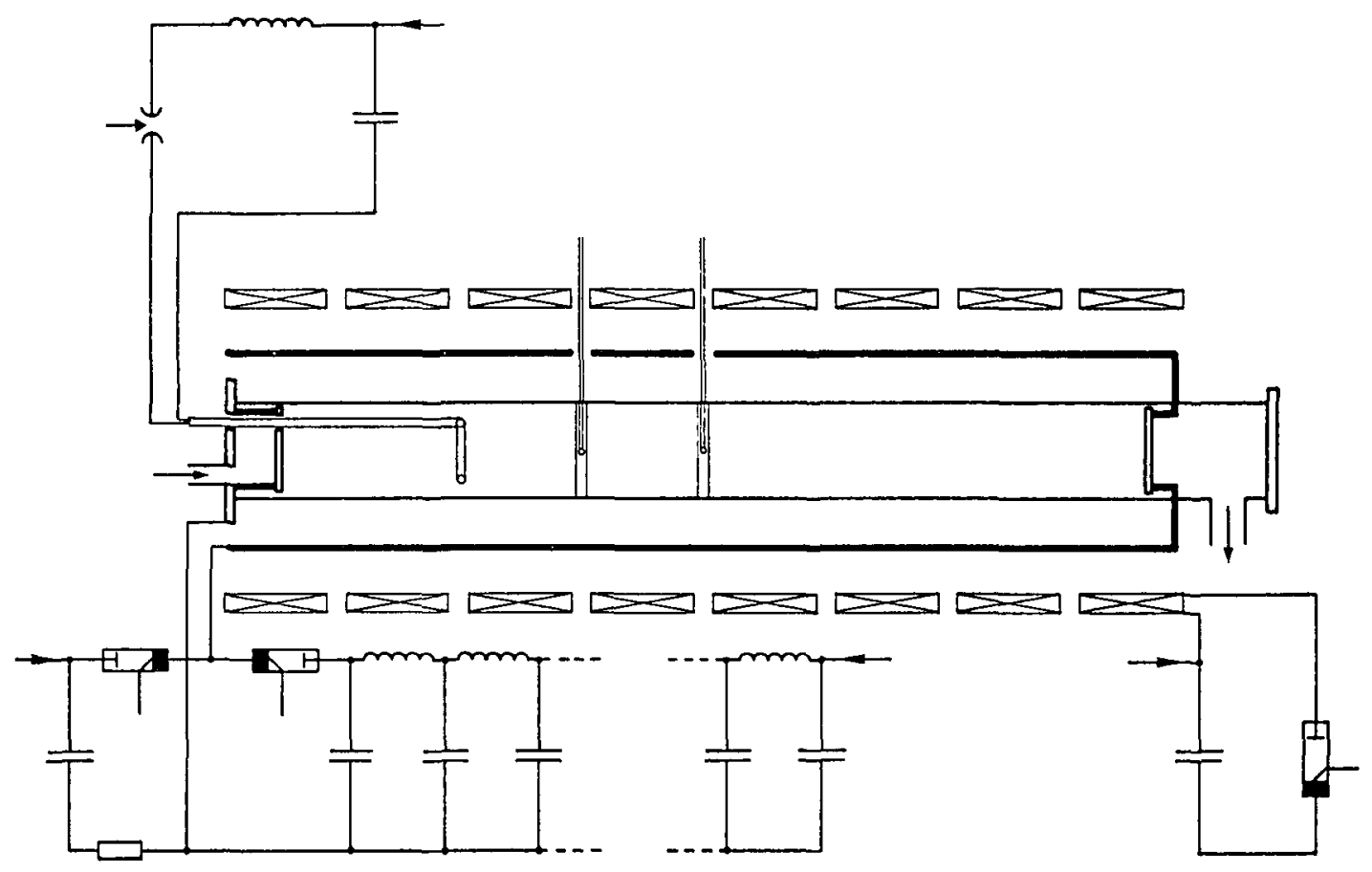

Figure 1. Schematic drawing of the experimental apparatus.

the left and to the right of $r=r^{\prime}$.† Since we can construct such a solution for each value of $k$ in the range given in (10), we have a continuous spectrum of eigenvalues.

At first glance, it might seem that, at a point where $D=0,(5)$ also has a singularity. However, this singularity is merely apparent. In fact, by means of (7), it can be shown that (5) is equivalent to a system of two first-order equations with no singularity at $D=0$.

Thus, we have the following distribution of eigenvalues of $k$. At a frequency above the lowest cut-off frequency, discrete modes and a continuous spectrum of modes should propagate, while below this frequency only continuous spectrum should be found. These modes carry all three components of the magnetic field.

\section{Experiment}

\subsection{Experimental apparatus}

The experimental apparatus consists of a linear pyrex glass tube $110 \mathrm{~cm}$ long and $9.4 \mathrm{~cm}$ dia., coaxial with a pulsed uniform $4.7 \mathrm{kG}$ magnetic field. The tube is filled with helium at a pressure 0.445 torr. Figure 1 is a schematic drawing of the apparatus. The magnetic field solenoid consists of 8 series-connected $32 \mathrm{~cm}$ dia. coils separated by insulating spacers. Switching a $12 \mathrm{~kJ}$ capacitor bank through the coils produces the magnetic field which has a period $4.4 \mathrm{~ms}$.

The plasma is prepared by discharging a $185 \mu \mathrm{F}$ capacitor bank, charged to

$\dagger$ Mathematical details of the matching of singular solutions across a singularity were given by Barston (1964). 


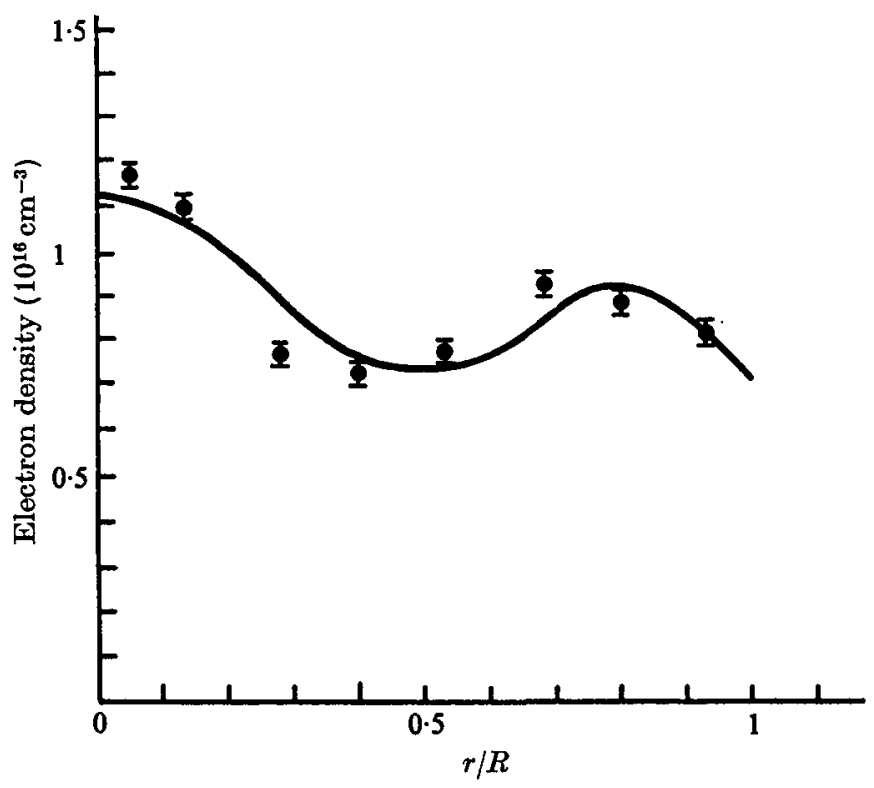

Figure 2. Electron density distribution.

$8 \mathrm{kV}$, between the two electrodes, $80 \mu$ s before the magnetic field reaches its peak value. The circuit is critically damped by a resistor. The current pulse rises to $77 \mathrm{kA}$ in $15 \mu$ s and is $55 \mu$ s wide. $60 \mu$ s after the plasma preparation was initiated, a current of $30 \mathrm{kA}$, stationary during $50 \mu \mathrm{s}$, is produced by switching on a $3 \mathrm{kV}$ unmatched pulse line.

The magnetohydrodynamic waves are generated, at peak magnetic field, by discharging a capacitor through a single-loop antenna positioned in one end of the tube. The plane of the loop is perpendicular to the axial magnetic field. A variable number of capacitors, connected to the antenna by a coaxial conductor, is used to provide the excitation frequencies over the range $0 \cdot 3-1 \cdot 1 \mathrm{Mc} \mathrm{s}^{-1}$. A $20 \mathrm{~cm}$ dia. brass shield, of suitable ratio of inductance to resistance, is positioned inside the solenoid to minimize the transformer-like coupling between the antenna and the solenoid. For the theoretical computations this shield determines the boundary condition at 'infinity' (i.e. $B_{r}$ vanishes on the shield).

The waves are detected by small magnetic probes which enter the plasma across a diameter of the discharge tube. The probes consist of three mutually perpendicular coils, the output from the two ends being subtracted using a differential amplifier. The velocity of the waves were determined by comparing the received signals from the two probes situated at $13 \mathrm{~cm}$ and $26 \mathrm{~cm}$ from the exciter, respectively. The same probes were also used for the determination of the values of the stationary magnetic field components.

Electron densities were determined using $\mathrm{He}-\mathrm{Ne}$ laser interferometer ( $\lambda=6328 \AA$ ), according to the method of Ashby \& Jephcott (1963). The measurements were performed at different radii along the lines parallel to the axis of the discharge tube. 


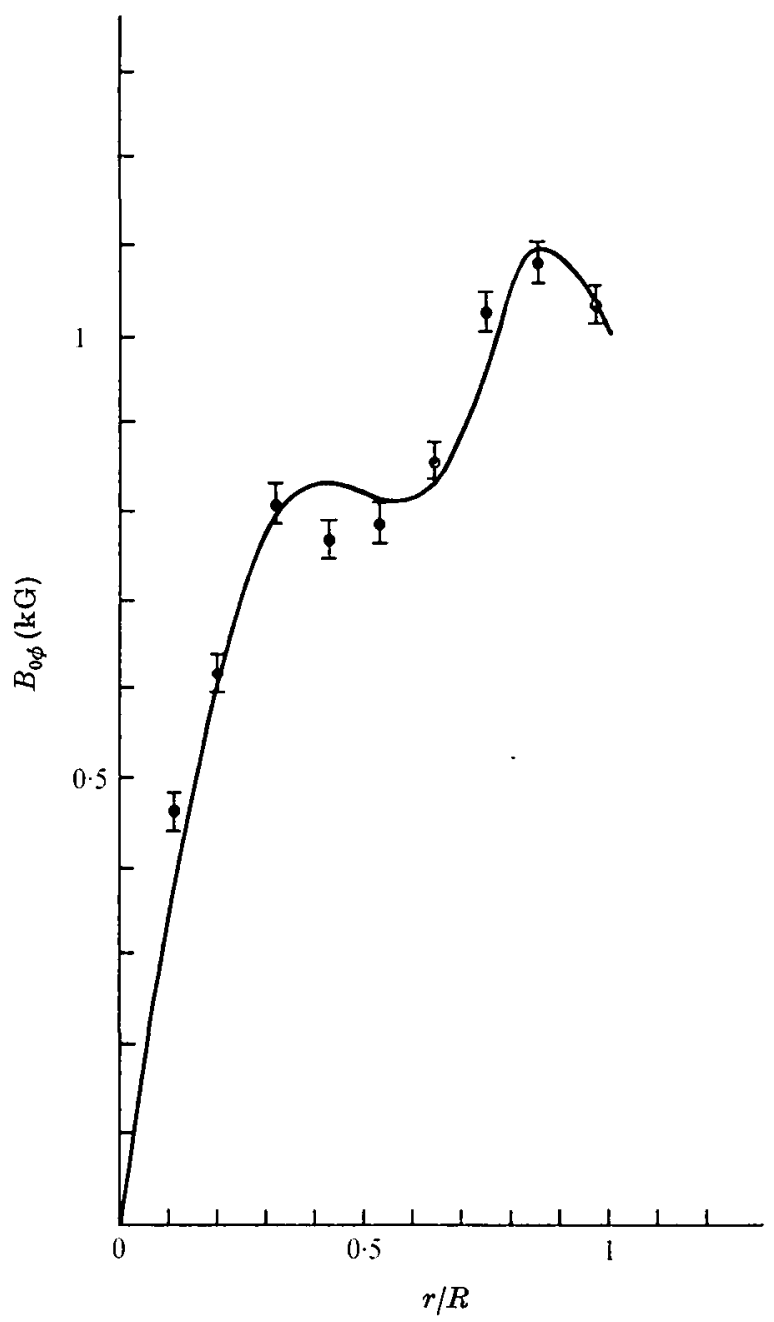

FIGURE 3. Profile of the azimuthal component of the stationary magnetic field.

\subsection{Results and discussion}

In order that the experimental results may be compared with the conclusions of the theory, it is necessary to determine the radial distribution of the plasma density and the profile of the azimuthal component of the stationary magnetic field. The results of the measurements are shown in figures 2 and 3 . The solid lines, plotted in these figures, are the polynomial fits used for the computations.

The dispersion relation of the excited waves was determined by measuring the velocity of the waves. For these measurements the probes were positioned on the axis of the discharge tube, and the axial component $B_{z}$ of the wave field was detected. The phase velocity is plotted as a function of frequency in figure 4. Here the solid line represents the theoretical curve corresponding to the lowest discrete mode of the compressional wave, and the dashed lines show the lower and upper limits of the continuous spectrum of modes of the torsional wave. From the experimental data we conclude that, above the cut-off frequency, the propa- 


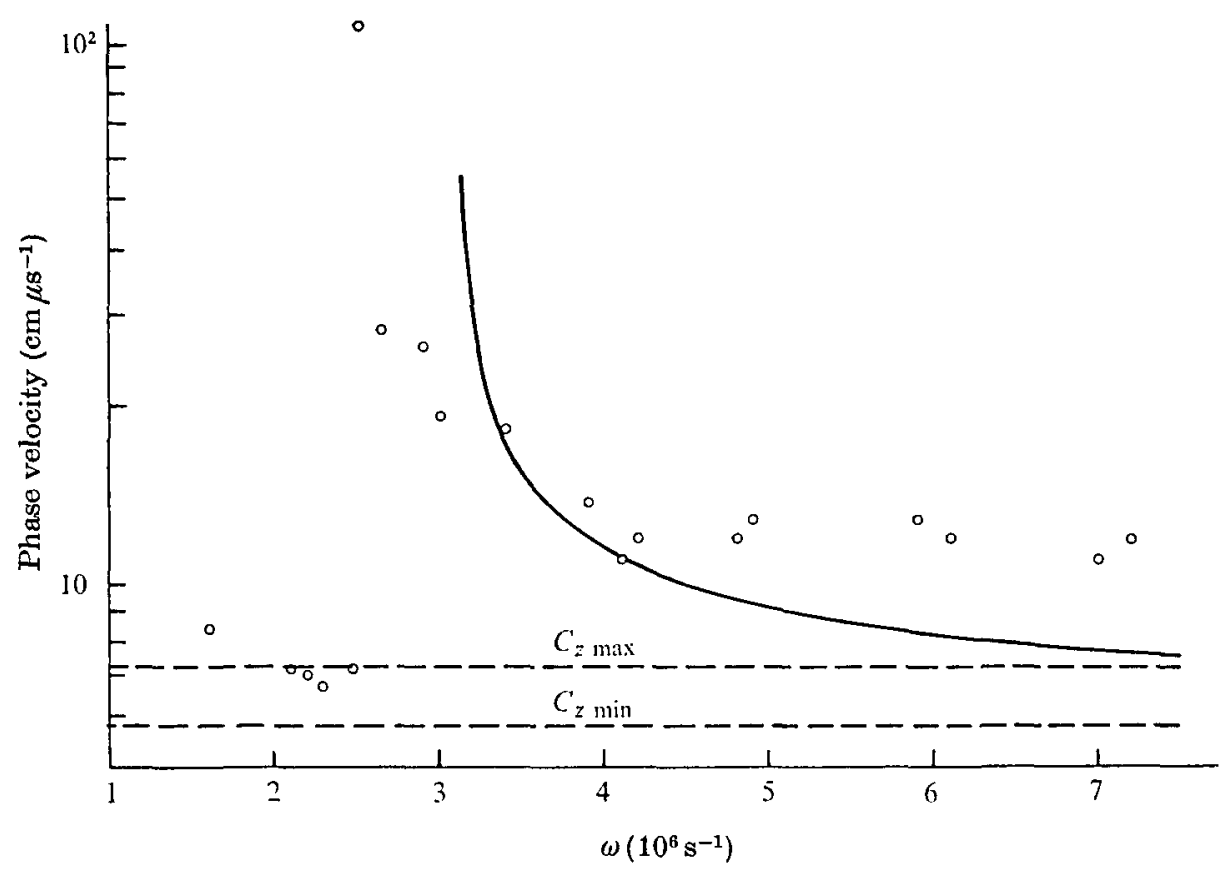

Figdre 4. Variation of phase velocity with frequency.

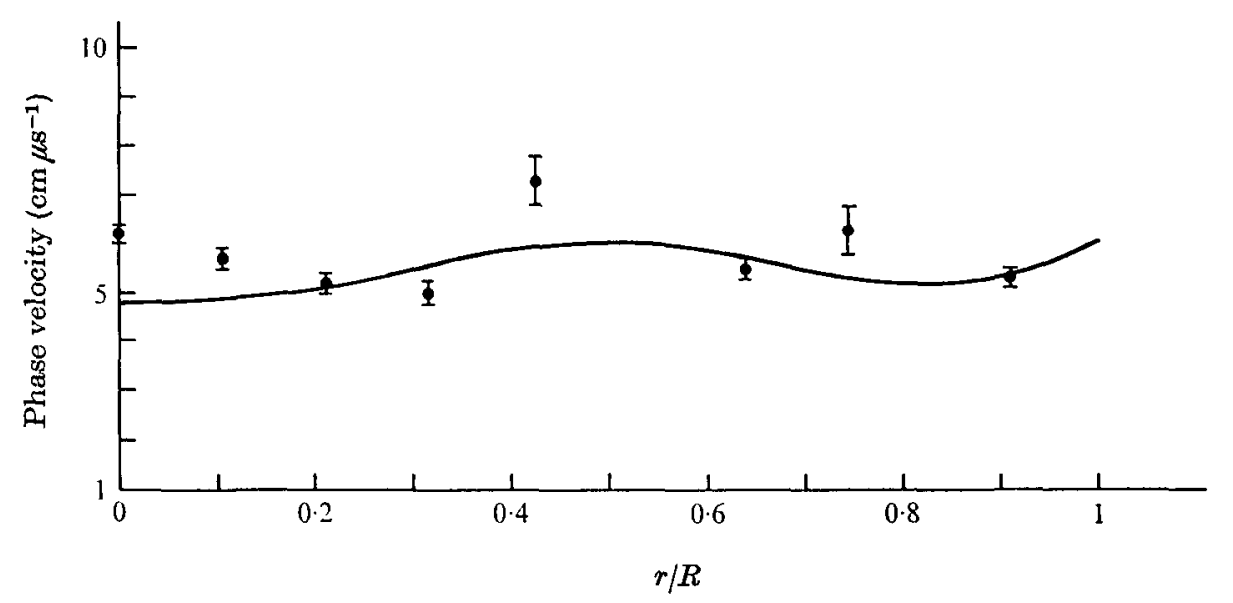

FIGURE 5. Variation of phase velocity with radius.

gating signal is predominately made of the discrete modes, while below this frequency the signal is only carried by the continuous spectrum of modes.

A significant property of the torsional wave is that the phase velocity varies with radius. Figure 5 gives the wave velocity against radius at a frequency of $0.33 \mathrm{Mc} \mathrm{s}^{-1}$, together with a graph of the function $C_{z}$ defined in (5). We see that the wave velocity does vary with radius, though the agreement with the theoretical velocity variation is not particularly close.

The radial profiles of the magnetic field components of the compressional wave were measured for the frequency of $0.45 \mathrm{Mcs}^{-1}$ at a distance of $13 \mathrm{~cm}$ from the 


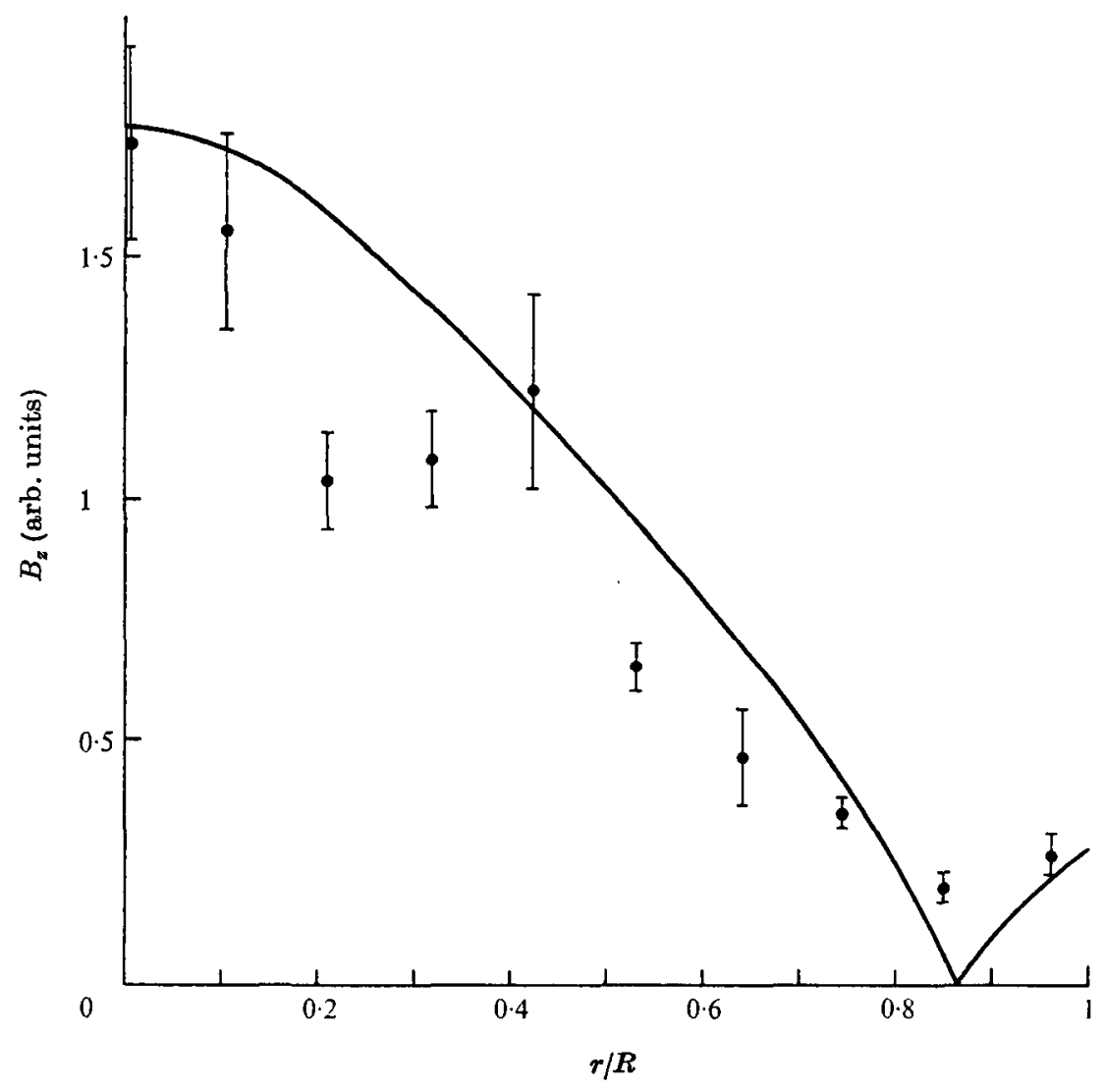

Fraure 6. Profile of the axial component of the wave magnetic field.

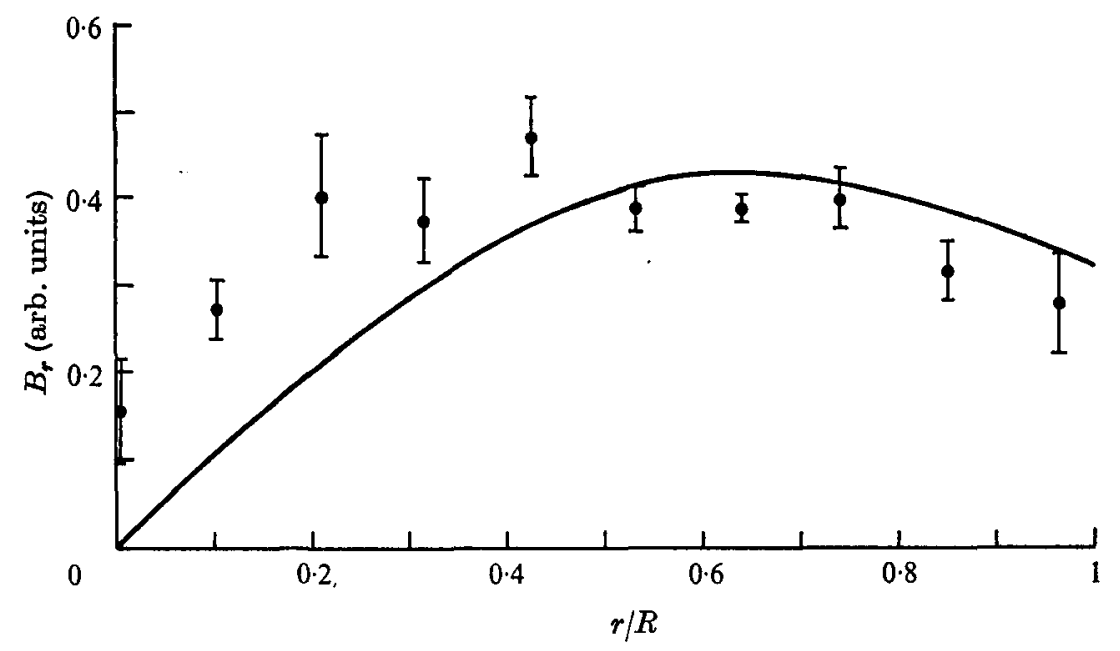

FIGURE 7. Profile of the radial component of the wave magnetic field. 


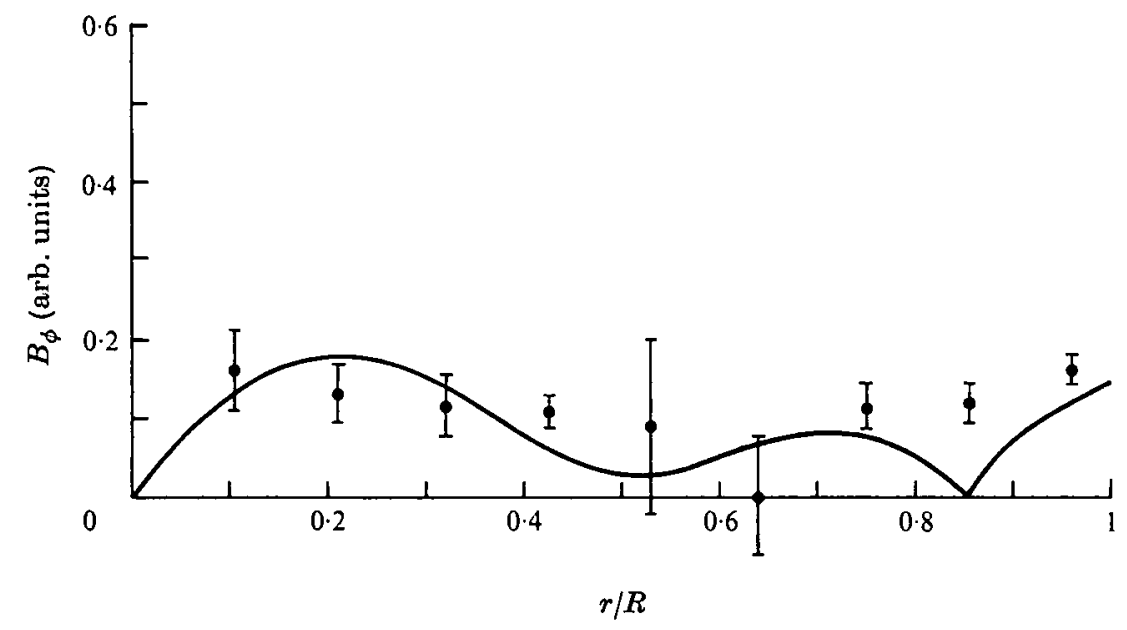

Figure 8. Profile of the azimuthal component of the wave magnetic field.

antenna. The results of these measurements are shown in figures 6-8, together with the theoretical profiles. Since the theoretical cut-off frequency occurs above the experimental one, the theoretical field profiles correspond to the theoretical frequency at which the wavelength was the same as that measured at $0.45 \mathrm{Mc} \mathrm{s}^{-1}$. We note, in particular, that the compressional mode carries an azimuthal component $B_{\phi}$ of the wave field, as was also observed by Müller et al. (1973).

Thus our results show that, in a radially non-uniform plasma column an axial current and a density gradient cause a coupling between the axisymmetric compressional and torsional waves.

The authors are grateful to Prof. O. Huber and Prof. H. Schneider for their continued interest in this work. The work was supported by the Swiss National Science Foundation.

\section{REFERENCES}

AshвY, D. E. \& JеРнсотт, D. F. 1963 Appl. Phys. Lett. 3, 13.

Barston, E. M. 1964 Ann. Phys. (N.Y.) 29, 282.

Cross, R. C. \& Lehane, J. A. 1968 Phys. Fluids, 11, 2621.

Davies, B. 1969 Phys. Rev. Lett. 22, 1246.

Kadomtsev, B. B. 1966 Reviews of Plasma Physics (ed. M. A. Leontovich), p. 2. New York: Consultants Bureau.

McPherson, D. A. \& Pridmore-Brown, D. C. 1966 Phys. Fluids, 9, 2033.

Morrow, R. \& Brennan, M. H. 1971 Plasma Phys. 13, 75.

MÜller, G., RÄUchle, E. \& Schüller, P. G. 1973 Plasma Phys. 15, 925.

Pneuman, G. W. 1965 Phys. Fluids, 8, 507. 